\title{
DETERMINACIÓN DEL NITRÓGENO POTENCIALMENTE MINERALIZABLE Y LA TASA DE MINERALIZACIÓN DE NITRÓGENO EN MATERIALES ORGÁNICOS
}

\section{DETERMINATION OF POTENTIALLY MINERALIZABLE NITROGEN AND THE RATE OF NITROGEN MINERALIZATION IN ORGANIC MATERIALS}

\author{
Aydee Figueroa-Barrera1, Javier G. Álvarez-Herrera², Andrés F. Forero ${ }^{3}$, César Salamanca ${ }^{4}$, \\ Lida P. Pinzón ${ }^{5}$
}

Recibido para publicación: Noviembre 8 de 2011 - Aceptado para publicación: Abril 9 de 2012

\begin{abstract}
RESUMEN
La aplicación de materiales orgánicos como fuente de fertilización es una práctica común en sistemas de producción limpia y orgánica; sin embargo, no se cuenta con los suficientes criterios técnicos para cuantificar la dosis adecuada. En este sentido, se presentan sobredosificaciones o subdosificaciones que pueden afectar la rentabilidad en el sistema productivo. Por tal razón, se utilizaron siete materiales orgánicos, comunmente utilizados en la sabana de Bogotá, tres derivados de residuos de cosecha (Compost de Rosas, Compost CIAA (CCIAA) y soil-aid $(\mathbb{)})$ y cuatro derivados de residuos animales (Gallinaza, Porquinaza, Lombrihumus y Conejaza). Se les determinó el nitrógeno potencialmente mineralizable y la tasa de mineralización utilizando el método de incubación. El diseño experimental utilizado fue completamente al azar, con ocho tratamientos (siete materiales orgánicos y un testigo), y tres repeticiones para un total de 24 unidades experimentales. La medición se realizó en intervalos semanales desde la semana uno hasta la semana nueve, después de la incubación. Los materiales derivados de residuos animales resultaron en general mejores que los provenientes de residuos vegetales en el proceso de mineralización de N, como es el caso de la conejaza que liberó 5197 $\mathrm{mg} \cdot \mathrm{kg}^{-1}$ de nitrógeno mineral, valor que representa el $20 \%$ del nitrógeno total inicial. El grado de ajuste del modelo logarítmico fue superior al $80 \%\left(r^{2}>0,80\right)$ en el $86 \%$ de los materiales evaluados.
\end{abstract}

Palabras claves: materia orgánica, carbono orgánico, amonio, nitrato, método Kjeldahl

\footnotetext{
${ }^{1}$ Ingeniero Agrónomo. Grupo de Investigaciones Agrícolas, Facultad de Ciencias Agropecuarias, Universidad Pedagógica y Tecnológica de Colombia, Tunja, Colombia.

${ }_{2 *}$ Profesor asistente, Grupo de Investigaciones Agrícolas, Facultad de Ciencias Agropecuarias, Universidad Pedagógica y Tecnológica de Colombia, Tunja. Colombia. E-mail: jgalvarezh@gmail.com

${ }^{3}$ Investigador Programa Suelos y Nutrición Vegetal. Centro de Investigaciones y Asesorías Agroindustriales, Universidad Jorge Tadeo Lozano. Bogotá. Colombia.

${ }^{4}$ Investigador Programa Suelos y Nutrición Vegetal Centro de Investigaciones y Asesorías Agroindustriales, Universidad Jorge Tadeo Lozano. Bogotá. Colombia.

${ }^{5}$ Ingeniera Agrónoma. Grupo de investigaciones agrícolas. Facultad de ciencias agropecuarias, Universidad Pedagógica y Tecnológica de Colombia, Tunja. Colombia.
} 


\begin{abstract}
The application of organic materials as fertilizers is a common practice in clean production and organic systems, however, there is not enough technical criteria to measure the right dose. In this sense, overdoses or underdosing may occurs affecting the profitability of the production system. For this reason, it was used seven organic materials, commonly used in the Sabana de Bogotá, three derived from crop residues (Sagaro Compost, Compost CIAA (ICCA), soil-aidß) and four derived from animal waste (chicken, pork, rabbit and ground lombris derived humus). It was determined its potentially mineralizable nitrogen and the rate of nitrogen mineralization using the incubation method. The experimental design was completely randomized with eight treatments (seven organic materials and a control), and three repetitions for a total of 24 experimental units. The measurement was performed at weekly intervals from week one to week nine, after incubation. Waste materials derived from animals were generally better than those from plant residues in the $\mathrm{N}$ mineralization process, as is the case of rabbit-derived humus that release $5.197 \mathrm{mg} \cdot \mathrm{kg}^{-1}$ of mineral nitrogen, a value that represents $20 \%$ of initial total Nitrogen. The degree of adjustment of logarithmic model was more than $80 \%$ (R2> 0.80 ) in $86 \%$ of the evaluated materials.
\end{abstract}

Key Words: organic, organic carbon, ammonium, nitrate, Kjeldahl method.

\section{INTRODUCCIÓN}

En la actualidad, los municipios aledaños a la sabana de Bogotá tienen sistemas de producción avícola y bovina que se caracterizan por tener una alta densidad de animales en pequeñas áreas, lo cual genera la producción de grandes cantidades de estiercol que sirve como materia prima para la preparación de abonos orgánicos, también existe disponibilidad de otros materiales orgánicos como desechos de cosecha, generalmente producidos en las mismas unidades agrícolas que presentan condiciones adecuadas para su uso en la agricultura (Forero et al. 2008).

El uso de estos materiales orgánicos en sistemas de producción agrícola es una práctica generalizada. En los cultivos de hortalizas en Cundinamarca el 83\% de los productores lo ha implementado (Forero y Escobar 2010) y se estima que la aplicación de gallinaza anualmente en sistemas de monocultivo de espinaca en el municipio de
Cota (Cundinamarca) se encuentra entre 25 y 40 t ha $^{-1}$ año-1 $^{-1}$ (Medina et al. 2005).

El nitrógeno es el elemento más indicado para valorar la calidad de un abono orgánico (Gómez 2000), porque (i) presenta altas posibilidades de ocasionar daño ambiental por pérdidas de amoniaco $\left(\mathrm{NH}_{3}^{+}\right)$por volatilización, de nitrato $\left(\mathrm{NO}_{3}^{-}\right)$por lixiviación y por la presencia de emisiones de oxido nitroso $\left(\mathrm{N}_{2} \mathrm{O}\right)$ (Grigatti et al. 2007) y (ii) este elemento no existe en la fracción mineral del suelo y su disponibilidad depende de la presencia de materia orgánica mineralizable y de los procesos de fijación biológica del $\mathrm{N}$ atmosférico, por las transformaciones que presenta (Mazarino 2002).

Forero et al. (2008) realizaron la caracterización de ocho materiales orgánicos con aplicación potencial para la producción de hortalizas en la sabana de Bogotá, en el que se determinó el perfil nutricional. En cuanto al contenido de nitrógeno, se destacaron, en respectivo 
orden, la conejaza y el compost de rosas, con contenidos que fluctuaron entre $2,0 \%$ y $2,5 \%$ de $\mathrm{N}$ total base seca, seguidas por los materiales porquinaza, soil-aid $(\mathbb{R}$ y gallinaza con valores entre $1,0 \%$ y $2,0 \%$ de $\mathrm{N}$ total con base seca. Con respecto a la relación carbono nitrógeno $(\mathrm{C} / \mathrm{N})$, esta fluctuó entre 11 y 15, lo que indica que todos los materiales orgánicos tienen suficiente nitrógeno para satisfacer las necesidades de los microorganismos degradantes.

Una de las desventajas que presentan los materiales orgánicos frente a los fertilizantes de síntesis química, es el desconocimiento del aporte real de nitrógeno en forma mineral, puesto que la disponibilidad de $\mathrm{N}$ después de la aplicación de un abono orgánico no puede ser estimada a partir del contenido de $\mathrm{N}$ total, sino que está condicionada por una serie de factores que afectan su liberación, ya sea retardándola o acelerándola.

Aproximadamente más del 95\% del $\mathrm{N}$ total que se encuentra en el suelo está en forma orgánica y éste es poco disponible para las plantas, pero al descomponerse por acción de los microorganismos, forma nitrógeno mineral asimilable por los cultivos y en este proceso conocido como mineralización, se distinguen dos etapas: la amonificación y la nitrificación (Rodríguez 2002). La amonificación es realizada por organismos quimiotróficos que requieren un periodo de tiempo para que el ciclo de descomposición se realice completamente, dependiendo del volumen de material orgánico añadido, el suministro de $\mathrm{N}$ utilizable, la proporción de fracciones lábiles y resistentes a la biodegradación, la temperatura y el nivel de humedad del suelo (Mactar 2007).
La nitrificación la realizan un conjunto bastante reducido de especies aerobias, bacterias muy sensibles a los agentes externos, ésta comprende dos fases, la primera es conocida como nitritación y en ella se oxida el amoniaco $\left(\mathrm{NH}_{3}\right)$ a nitrito $\left(\mathrm{NO}_{3}\right)$ por nitrosobacterias. La segunda fase se conoce como nitratación y se oxida el $\mathrm{NO}_{3}$ a $\mathrm{NO}_{2}$ por las nitrobacterias (Navarro y Navarro 2000). Tanto la nitrificación como la mineralización del nitrógeno orgánico en el suelo, se ven afectadas por la humedad, la temperatura y factores químicos entre los que se encuentra el $\mathrm{pH}$, las sales y la presencia de compuestos inorgánicos y organismos tóxicos (Sikora y Szmidt 2004), así como también por la relación $\mathrm{C} / \mathrm{N}$, presencia de sustancias aleloquímicas de las plantas y el aporte de nutrientes esenciales (Vernimmen et al. 2007).

La relación $\mathrm{C} / \mathrm{N}$ se considera como la información más sencilla sobre la capacidad de mineralización de un material orgánico, ya que los contenidos de carbono y nitrógeno, son esenciales para la vida y la reproducción de los microorganismos. Cuando la relación $\mathrm{C} / \mathrm{N}$ es menor de 20 se presentará una mayor velocidad y magnitud de descomposición, por el contrario si la relación $\mathrm{C} / \mathrm{N}$ es mayor de 25 el proceso de descomposición es lento lo que genera inmovilización de $\mathrm{N}$ por parte de los microorganismos (Feral et al. 2003; Cerrato et al. 2007).

Conocer la relación $\mathrm{C} / \mathrm{N}$ de los materiales orgánicos no es suficiente para determinar la cantidad de $\mathrm{N}$ que se mineraliza, entonces se requiere determinar otra serie de características, como el potencial y la tasa de mineralización de $\mathrm{N}$. El $\mathrm{N}$ potencialmente mineralizable es la máxima cantidad de $\mathrm{N}$ que 
puede ser liberado de un material orgánico después de su descomposición por acción de los microorganismos (Cerrato et al. 2007), este potencial se establece a partir de la mineralización acumulada, la cual se define como la cantidad de $\mathrm{N}$ liberado en un periodo de tiempo específico (Brady y Weil 1999).

La tasa de mineralización de $\mathrm{N}$ es la velocidad o grado de mineralización y se interpreta como el porcentaje de $\mathrm{N}$ que se mineraliza y que permite conocer cuanto está siendo liberado en forma disponible en un periodo de tiempo determinado (Cerrato et al. 2007). González et al. (1995) mencionan que el contenido óptimo de humedad para la mineralización es $75 \%$ de la capacidad de retención de humedad del material orgánico y Benítez et al. (1998) mencionan que a $30{ }^{\circ} \mathrm{C}$ se obtiene una mayor velocidad de mineralización.

Por lo anterior, el objetivo de esta investigación fue determinar el $\mathrm{N}$ potencialmente mineralizado y la tasa de mineralización de algunos materiales orgánicos comunes en la Sabana de Bogotá, con el fin de conocer la cantidad de nitrógeno disponible en cada material orgánico y correlacionarlo con otras propiedades de los mismos, buscando nuevas alternativas de fertilización para los diferentes sistemas de producción existentes.

\section{MATERIALES Y MÉTODOS}

La investigación fue desarrollada en el Centro de Bio-Sistemas de la Universidad de Bogotá Jorge Tadeo Lozano con sede en el Municipio de Chía, departamento de Cundinamarca.

Se utilizaron tres materiales orgánicos de origen vegetal (compost de rosas, soil-aid $(\mathbb{B}$ y compost del Centro de Bio-Sistemas (CIAA)) y cuatro de origen animal (porquinaza, gallinaza, lombrihumus y conejaza), generalmente fabricados en las mismas unidades de producción de los municipios aledaños a la sabana de Bogotá, y que presentan condiciones adecuadas para el uso en la agricultura. Estos fueron analizados empleando el método de incubación propuesto por Stanford y Smith (1972).

Los materiales compostados fueron analizados bajo un diseño completamente al azar, con ocho tratamientos, siete materiales orgánicos y un testigo (arena), con tres repeticiones para un total de 24 unidades experimentales (en la Tabla 1 se describe los tratamientos con sus respectivas características químicas).

Tabla 1. Caracterización química de los tratamientos

\begin{tabular}{|c|c|c|c|c|c|}
\hline Material orgánico & Tratamiento & $\mathrm{CO}^{1}(\%)$ & $\mathrm{MO}^{2}(\%)$ & $C / N^{3}(p: p)$ & N Total ${ }^{4}(\%)$ \\
\hline Compost de Rosas & $\mathrm{T} 1$ & 29,1 & 63,1 & 13,2 & 2,2 \\
\hline Porquinaza & $\mathrm{T} 2$ & 19,1 & 41,4 & 11,9 & 1,6 \\
\hline Soil-aid $(\mathbb{R}$ & T3 & 18,3 & 39,7 & 10,2 & 1,8 \\
\hline Gallinaza & $\mathrm{T} 4$ & 15,3 & 33,2 & 12,8 & 1,4 \\
\hline Compost CIAA & T5 & 11,6 & 25,2 & 15,4 & 1,0 \\
\hline Conejaza & T6 & 26,5 & 57,5 & 10,6 & 2,5 \\
\hline Lombrihumus & T7 & 12,2 & 26,4 & 16,3 & 1,0 \\
\hline Arena & T8 & 0,4 & 0,9 & 1,1 & 0,4 \\
\hline
\end{tabular}

${ }^{1}$ Carbono orgánico; ${ }^{2}$ Materia Orgánica; ${ }^{3}$ Relación Carbono Nitrógeno; ${ }^{4}$ Porcentaje de nitrógeno base seca. 
Inicialmente, se determinó la humedad de cada muestra para expresar los resultados con base en masa seca, luego cada uno de los materiales fue secado al aire y a la sombra, se molieron y tamizaron a través de una malla de $2 \mathrm{~mm}$. Posteriormente se procedió a colocar 25 g de cada material en tubos de lixiviación de $200 \mathrm{~mL}$ previamente recubiertos en la parte inferior con papel filtro, lana de vidrio y $10 \mathrm{~g}$ de arena lavada con cloruro de potasio $(\mathrm{KCl})$.

Los tubos de lixiviación se mantuvieron durante nueve semanas en una incubadora a una temperatura constante de $32{ }^{\circ} \mathrm{C}$ y humedad del $60 \%$ y $70 \%$, con toma de muestras semanal. Con el fin de mantener húmedas las muestras y simular condiciones favorables para los microorganismos, se adicionó semanalmente $12,5 \mathrm{~mL}$ de una solución nutritiva compuesta por Sulfato de magnesio $\left(\mathrm{MgSO}_{4} 0,002 \mathrm{M}\right)$, fosfato de calcio $\left(\mathrm{Ca}\left(\mathrm{HPO}_{4}\right)_{2} \mathrm{H}_{2} \mathrm{O} \quad 0,007 \mathrm{M}\right)$ y Sulfato de potasio ( $\left.\mathrm{K}_{2} \mathrm{SO}_{4} 0,0025 \mathrm{M}\right)$, se excluyó el $\mathrm{N}$ para evitar alteraciones en los contenidos de este elemento proveniente de los materiales evaluados.

El nitrógeno mineral inicialmente presente en las muestras fue lavado con $100 \mathrm{~mL}$ de $\mathrm{CaCl}_{2}$ 0,01 M (Stanford y Smith 1972), seguido de la adición de 12,5 mL de solución nutritiva. Este mismo procedimiento se realizó después de cada lixiviación dentro del período de incubación.

Para la determinación de la concentración de nitrógeno se tomó una alícuota de $10 \mathrm{~mL}$ de solucion procedente del extracto de los materiales orgánicos, de acuerdo al método Kjeldahl mencionado por Bremner y Keeney (1965), empleado para cuantificar el $\mathrm{N}$ mineral. Este método determina el $\mathrm{N}$ mineral, basado en la extracción del amonio intercambiable por equilibrio de la muestra de suelo con $\mathrm{KCl} 1 \mathrm{~N}$ y su determinación por destilación mediante arrastre de vapor en presencia de MgO. La adición de la aleación de Devarda permite incluir la determinación de nitratos, convirtiéndolo en amonio por reducción (Martinez 2010). Posteriormente, el destilado fue recibido en ácido bórico y titulado con ácido sulfúrico normalizado. Luego se procedió a valorar el nitrógeno mineralizado en forma amoniacal y nítrica $\left(\mathrm{N}-\mathrm{NH}_{4}{ }^{+}\right.$y $\left.\mathrm{N}-\mathrm{NO}_{3}{ }^{-}\right)$, con el fin de conocer el nitrógeno total.

Para las variables evaluadas se realizó un análisis de varianza (Anova) entre tratamientos y entre semanas. Se usó el software estadístico SAS ${ }$ v. 8.1e (SAS Institute Inc., Cary, NC). Para las variables que mostraron diferencias significativas, se efectúo la prueba de Duncan al 95\%.

\section{RESULTADOS Y DISCUSIÓN}

\section{Nitrógeno potencial mineralizable}

Se encontraron diferencias estadísticas altamente significativas entre tratamientos $y$ semanas para el nitrógeno potencialmente mineralizable. En el período de incubación los materiales orgánicos con mayor $\mathrm{N}$ potencialmente mineralizable fueron conejaza con $5179 \mathrm{mg} \mathrm{kg}^{-1}$, compost de rosas (sagaro) con $3104 \mathrm{mg} \mathrm{kg}^{-1}$ y porquinaza con 2821 $\mathrm{mg} \mathrm{kg}{ }^{-1}$, estos valores son altos con respecto

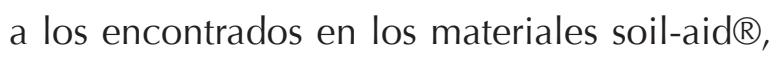
compost del CIAA y gallinaza, los cuales oscilaron entre 1000 y $1300 \mathrm{mg} \mathrm{kg}^{-1}$, que son cantidades apreciables pero no representan más del $20 \%$ del $N$ total que tenían los materiales (Figura 1). No obstante, todos los valores encontrados para los materiales orgánicos 


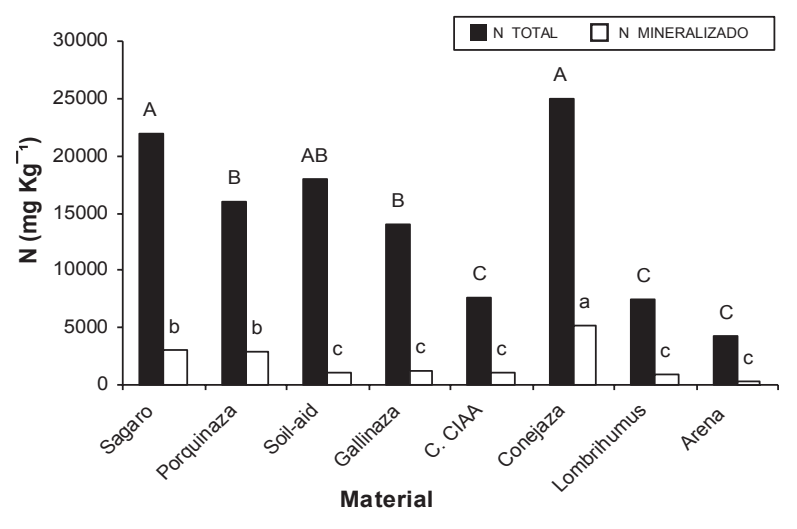

Figura 1. Nitrógeno total y mineralizado de siete materiales orgánicos. Sagaro (Compost de rosas). Promedios con letra en común no presentan diferencias significativas entre semanas según la prueba de Duncan $(P>0,05)$.

evaluados son superiores a los reportados para nitrógeno potencialmente mineralizable en suelos minerales por Benintende et al. (2003), lo que implica que cualquiera de los materiales que se aplique aportará una cantidad apreciable de $\mathrm{N}$ al suelo.

Completadas nueve semanas de incubación, se observa que las cantidades de nitrógeno mineralizado en la porquinaza y lombrihumus, son muy pequeñas en las últimas semanas y terminan de una manera asintótica, igualmente ocurrió con soil-aid $($ Figura 2). Este comportamiento muestra que estos materiales estaban constituidos por componentes lábiles o fácilmente mineralizables, lo que permitió la liberación del nitrógeno fácilmente mineralizable en las primeras semanas, el cual podría considerarse como realmente aprovechable por el cultivo. Del mismo modo, Benintende et al. (2007) mencionan que la mayor cantidad de $\mathrm{N}$ fácilmente mineralizado se obtuvo en incubaciones anaeróbicas de 7 días, y que los valores obtenidos permitieron establecer diferencias entre suelos y manejos diferentes.
Los materiales compost de rosas y conejaza no tuvieron una clara disminución en las cantidades de nitrógeno mineralizado en las últimas semanas, lo que se puede atribuir a que estos materiales presentaban componentes más resistentes al ataque de la biomasa microbiana. Para estos dos materiales, se puede afirmar que las nueve semanas de incubación no fueron suficientes para liberar todo el nitrógeno fácilmente mineralizable como se observó en los otros materiales. Además, en el caso de ser usados como enmienda de fertilización, los primeros materiales orgánicos que presentaron alta liberación podrían aplicarse como una herramienta de rápido efecto.

\section{Nitrógeno Nítrico $\left(\mathrm{NO}_{3}{ }^{-}\right)$}

Deacuerdo al análisis estadístico, se encontraron diferencias estadísticas altamente significativas $(P>0,0001)$ entre tratamientos y entre semanas para los $\mathrm{NO}_{3}^{-}$. En la semana dos se presentó la mayor liberación de $\mathrm{N}-\mathrm{NO}_{3}^{-}$, por parte de todos los materiales orgánicos (Figuras 2A y $2 \mathrm{~B})$, lo que indica la presencia de $\mathrm{N}$ fácilmente disponible, que al ser aplicado al suelo, probablemente tendrá un efecto inmediato.

El valor de $\mathrm{NO}_{3}{ }^{-}$presentó diferencias altamente significativas entre tratamientos, siendo la conejaza, el material de origen animal que presentóel mayor promediodurantelas primeras cinco semanas (Figura 2A). El compost de rosas fue el material con mayor liberación de nitratos en el caso de los materiales provenientes de residuos de cosecha (Figura $2 \mathrm{~B}$ ). A partir de la cuarta semana, a excepción de la conejaza y la porquinaza, los demás materiales presentaron una liberación de nitratos muy baja, lo cual pudo ser ocasionado por la inmovilización producida por las bacterias nitrificantes, o 
sencillamente porque el material no puede mineralizar más nitrógeno, pues este hace parte ya de la composición estructural del mismo. En cuanto a los materiales de origen vegetal, soilaid ${ }^{\circledR}$ y C.CIAA fueron los de menor liberación de nitratos.

\section{Nitrógeno Amoniacal $\left(\mathrm{N}-\mathrm{NH}_{4}{ }^{+}\right)$}

Se identificaron diferencias estadísticas significativas entre tratamientos para cada semana de incubación, presentando la porquinaza la mayor media en las primeras tres semanas (Figura 2C), liberando altos valores de $\mathrm{N}-\mathrm{NH}_{4}{ }^{+}$con respecto a otros materiales de origen animal (gallinaza, lombrihumus y conejaza) y a los provenientes de residuos vegetales (Compost de rosas, soil-aid® y C.CIAA).

Se observó que durante las primeras semanas de incubación, la mineralización del nitrógeno fue más rápida, (Figura 2C y 2D) debido a que en este periodo se produce la descomposición de azúcares, proteínas y celulosas, haciéndose más lenta hacia las últimas semanas en donde se están mineralizando aquellos materiales más resistentes y que necesitan más tiempo para descomponerse como lo reportan Rodríguez y Matus (1989) y Levi-Minzi et al. (1990).
La descomposición de los tejidos vegetales y la liberación y mineralización de $N$ frecuentemente muestran una fase inicial rápida, en la cual los tejidos son degradados por los microorganismos que componen la biomasa del suelo, obteniendo productos secundarios (constituyentes de la pared celular, como celulosa y hemicelulosa). Esta nueva biomasa y sus productos metabólicos son, a su vez, sustratos para la segunda fase, que es mucho más lenta, regulada principalmente por el contenido de lignina (Acero y Olaya 2006). En este estudio se identifica un período comprendido entre la segunda y la cuarta semana para la mayoría de los tratamientos en los cuales ocurrió este fenómeno.

En cuanto al porcentaje de nitrógeno mineralizado por semana (Tabla 2), se observa que hasta la semana tres, los materiales habían mineralizado más del 50\% del total mineralizable y se destacan los

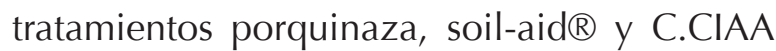
los cuales acumularon el $80 \%$ o más de la mineralización ocurrida en nueve semanas. En el caso de los demás materiales, se observa que la conejaza y el lombrihumus acumularon el $80 \%$ mineralizado en la semana cinco.

Tabla 2. Porcentaje de nitrógeno mineralizado de materiales orgánicos por semana.

\begin{tabular}{crrrrrrrrr}
\hline \multirow{2}{*}{ Semana } & \multicolumn{10}{c}{$\mathbf{N}(\mathbf{\%})$} \\
\cline { 2 - 9 } & $\begin{array}{c}\text { Compost } \\
\text { de rosa }\end{array}$ & C.CIAA & Soil-aid $\mathbb{R}$ & Gallinaza & Porquinaza & Conejaza & Lombrihumus & Arena \\
\hline 1 & 15,59 & 39,25 & 45,72 & 33,74 & 23,31 & 11,62 & 30,09 & 11,76 \\
2 & 44,99 & 71,45 & 82,32 & 60,32 & 64,78 & 38,93 & 54,77 & 40,83 \\
3 & 53,04 & 80,86 & 83,98 & 79,34 & 84,89 & 60,84 & 67,97 & 55,13 \\
4 & 57,53 & 84,29 & 86,91 & 85,99 & 91,54 & 73,41 & 77,78 & 66,03 \\
5 & 62,39 & 89,60 & 90,41 & 89,57 & 95,89 & 83,60 & 83,30 & 77,83 \\
6 & 69,04 & 93,78 & 93,85 & 93,17 & 97,44 & 88,07 & 88,86 & 86,41 \\
7 & 79,14 & 95,60 & 96,15 & 95,44 & 98,71 & 92,71 & 94,72 & 92,01 \\
8 & 90,68 & 97,42 & 98,02 & 97,25 & 99,45 & 96,38 & 96,94 & 92,48 \\
9 & 100,00 & 100,00 & 100,00 & 100,00 & 100,00 & 100,00 & 100,00 & 100,00 \\
\hline
\end{tabular}

C.CIAA: Compost del centro de Bio-Sistemas. 
Bajo las condiciones de la investigación, el nitrógeno de los materiales porquinaza, soilaid ${ }^{\circledR}$, gallinaza y $C . C I A A$ pasa rápidamente a formas disponibles, mientras que los materiales compost de rosas, conejaza y lombrihumus se mineralizan de una forma más gradual. Esto coincide con los análisis reportados por Galvis y Hernández (2004), donde en el $82 \%$ de las

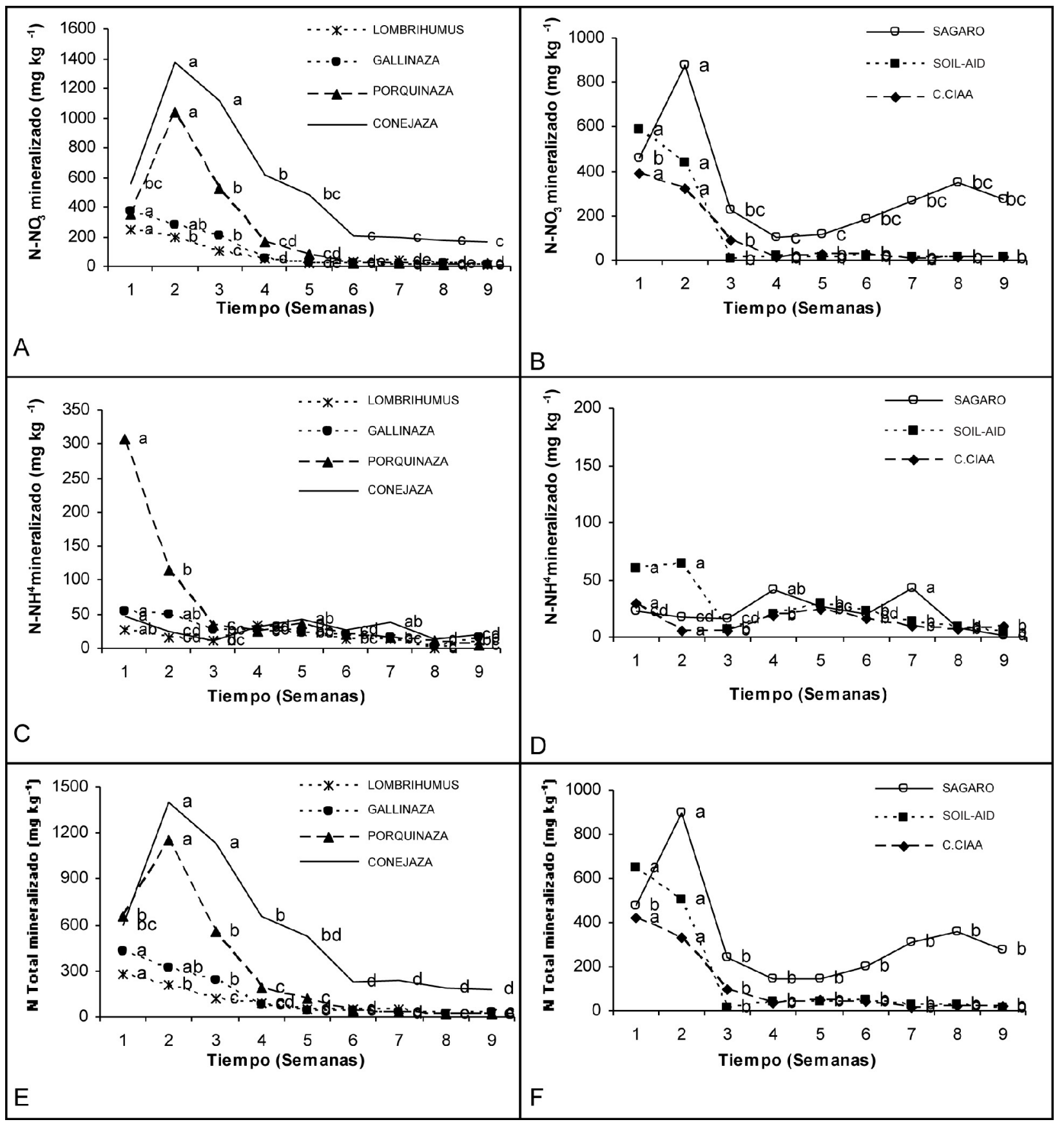

Figura 2. Mineralización de nitrógeno de materiales orgánicos. A) $\mathrm{N}-\mathrm{NO}_{3}-$ de materiales orgánicos de origen animal; B) $\mathrm{N}-\mathrm{NO}_{3}$ - de materiales orgánicos de origen vegetal; C) $\mathrm{N}-\mathrm{NH}_{4}{ }^{+}$de materiales orgánicos de origen animal; D) $\mathrm{N}-\mathrm{NH}_{4}{ }^{+}$de materiales orgánicos de origen vegetal; $\left.\mathrm{E}\right) \mathrm{N}$ total de materiales orgánicos de origen animal; F) $\mathrm{N}$ total de materiales orgánicos de origen vegetal. Promedios con letra en común no presentan diferencias significativas entre semanas según la prueba de Duncan $(P>0,05)$. Sagaro: compost de rosas. 
62 muestras el $\mathrm{N}$ mineralizado acumulado a la penúltima extracción alcanzó entre el 90\% y $100 \%$ del acumulado a la última extracción realizada.

\section{Tasa de Mineralización}

El Nitrógeno mineralizado en todos los materiales, se ajustó a un modelo logarítmico de la forma $y=\left(a^{*} \operatorname{Ln}(x)+b\right)$ (Figura 3$)$. El modelo logarítmico se ajustó con una alta correlación a las cantidades de $\mathrm{N}$ mineralizado acumuladas de los materiales provenientes de residuos de cosechas y los provenientes de desechos animales.

El material con mayor tasa de mineralización fue la conejaza con una pendiente relativa de $1183 \mathrm{mg} \mathrm{kg}^{-1}$ por semana, seguido de los

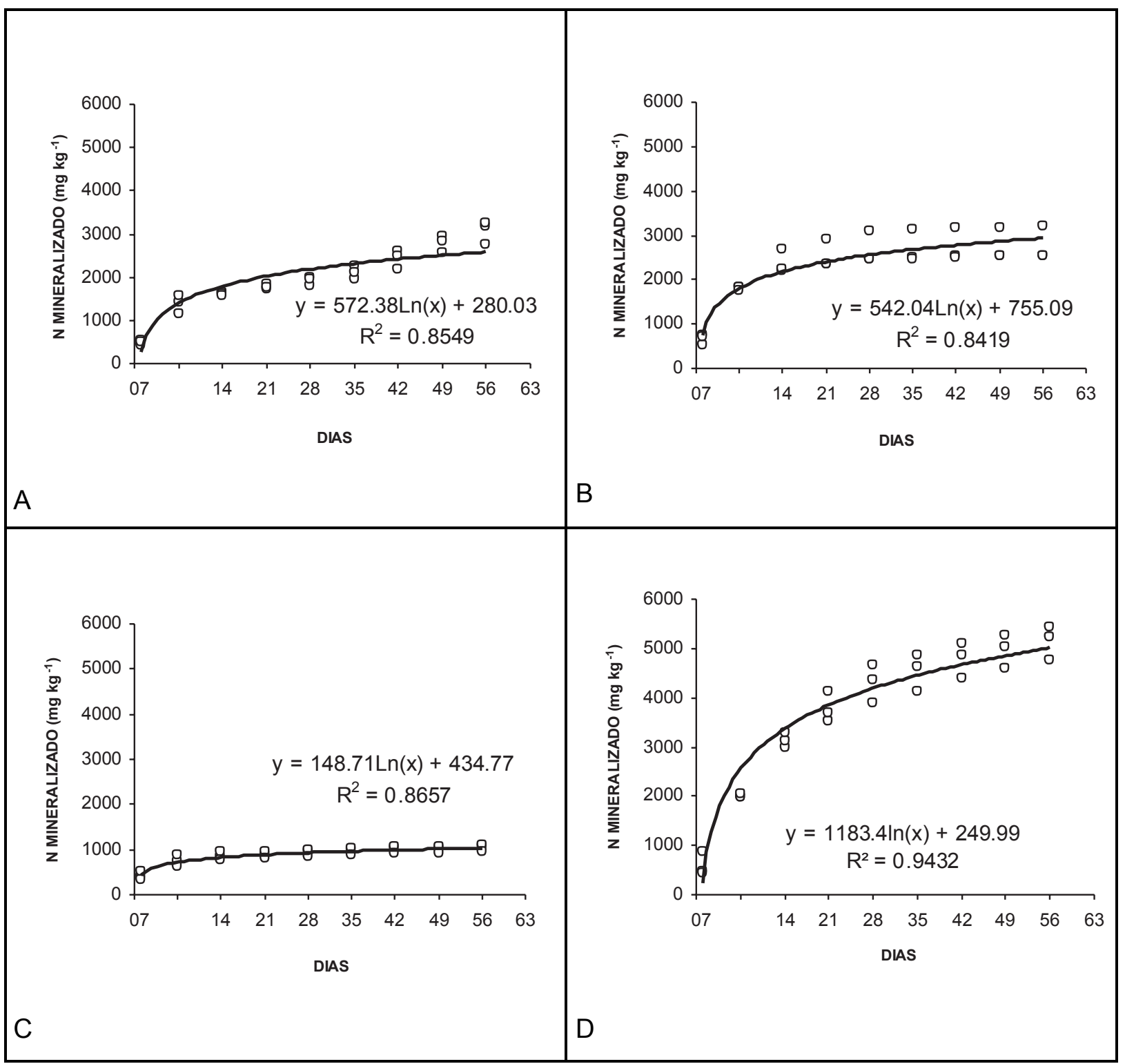

Figura 3. Modelos de mineralización de nitrógeno de A) Compost de rosas (Sagaro); B) Porquinaza; C) Compost del Centro de Bio-Sistemas (C.CIAA); D) Conejaza; mediante el método de incubación. 
materiales compost de rosas y porquinaza, los cuales presentaron valores de pendiente relativa de 572 y $543 \mathrm{mg} \mathrm{kg}^{-1}$ semana respectivamente. Los demás materiales presentaron bajas tasas de mineralización siendo la menor tasa la del compost CIAA con un 148,7 $\mathrm{mg} \mathrm{kg}^{-1}$.

El grado de ajuste del modelo exponencial fue superior al $80 \%\left(r^{2}>0,80\right)$ en seis de los siete materiales analizados. Esta observación indica que al tener el modelo logarítmico con un buen grado de ajuste, la confiabilidad del mismo para predecir la tasa de mineralización de $\mathrm{N}$ es más confiable.

\section{CONCLUSIONES}

La conejaza presentó los mayores contenidos de $\mathrm{N}$ mineralizable. Los materiales orgánicos provenientes de residuos de animales resultaron en general mejores que los provenientes de residuos vegetales en el proceso de mineralización de $\mathrm{N}$. El nitrógeno de los materiales porquinaza, soil-aid $\AA$, gallinaza y C.CIAA (compost del centro de Bio-Sistemas) pasa rápidamente a formas disponibles, mientras que los materiales compost de rosas, conejaza y lombrihumus se mineralizan de una forma más gradual.

\section{AGRADECIMIENTOS}

Los autores expresan su agradecimiento al Centro de Bio-Sistemas de la Universidad Jorge Tadeo Lozano por el apoyo brindado para la ejecución y desarrollo del presente trabajo, así, como al Ministerio de Agricultura y Desarrollo rural por el apoyo financiero.

\section{REFERENCIAS}

Acero, P. y Olaya, W. 2006. Establecimiento de las bases para definir las series de mineralización del carbono, nitrógeno y fósforo en tres materiales compostados. Tesis Ingeniero agrónomo, Universidad Nacional de Colombia, Bogotá.

Benintende, M., Múller, C., Benintende, S. y Sterren, M. 2003. Índice del potencial de mineralización de nitrógeno en los suelos. Revista Científica Agropecuaria 7(1):51-55.

Benintende, M., Benintende, S., Sterren, M., Musante, C., Saluzzio, M. y De Battista, J. 2007. Modelo para estimar la capacidad de aporte de nitrógeno del suelo. Agriscientia 24(2):97-101.

Benítez, C., Tejada, M. y González, J. 1998. Influencia de condiciones edafoclimáticas en la mineralización de $\mathrm{N}$-ureico en suelos mediterráneos. XVI Congreso Mundial de la Ciencia del Suelo. Montpellier, Francia. p243.

Brady, N. y Weil, R. 1999. The Nature and Properties of Soils, Prentice Hall, New Jersey, US. p881.

Bremner, J. y Keeney, D. 1965. Steam distillation methods for determination of ammonium, nitrate and nitrite. Annal. Chem. Acta 32:485-495.

Cerrato, M., Leblanc, H. y Kameko, C. 2007. Potencial de mineralización de nitrógeno de bokashi, compost y lombricompost 
producidos en la Universidad EARTH. Revista Tierra Tropical 3(2):183-197.

Feral, C., Epstein, H., Otter, L., Aranibar, J., Shugart, J., Macko, S. y Ramontshoz, J 2003. Carbon and nitrogen in the soilplant system along rainfall and landuse gradients in southern Africa. Arid Environments 54:327-343.

Forero, A., Escobar, H. y Medina, A. 2008. Caracterización de materiales orgánicos con aplicación potencial en la producción de hortalizas de hoja y brasicas en la Sabana de Bogotá. XIV Congreso Colombiano de la Ciencia del Suelo. Villavicencio, Octubre de 2008, p81.

Forero, A. y Escobar, H. 2010. Effect of conventional and organic vegetable production systems on soil chemical properties in the Bogotá Plateau (Colombia). Agronomía Colombiana 28(3):421-427.

Galvis-Spinola, A. y Hernández-Mendoza, T. 2004. Cálculo del nitrógeno potencialmente mineralizable. INCI 29(7):377-383.

Gómez, J. 2000. Abonos orgánicos. Impresora Feriva S. A. Colombia, p49-69.

González, S., Carballas, M. y Villar, M. 1995. Organic nitrogen mineralization in temperate humid-zone soils after two and six weeks of aerobic incubation. Biology and Fertility of Soils 20(4):237-242.
Grigatti, M., Perez, M., Blokc, W., Ciavattaa, C. y Veeken, A. 2007. A standardized method for the determination of the intrinsic carbon and nitrogen mineralization capacity of natural organic matter sources. Soil Biology \& Biochemistry 39(7):1493-1503.

Levi- Minzi, R., Riffaldi, R. y Saviozzi, A. 1990. Carbon mineralization in soil amended with different organic materials. Agriculture, Ecosystems and Enviroment 31(4): 325-335.

Mactar, S. 2007. Atividade e funcionalidade das comunidades nitrifcadoras, desnitrificadotas e fixadoras de nitrogênio em solos sob diferentes coberturas vegetais na região norte do estado do Rio de Janeiro. Tesis Doutorado Agronomía, Universidade Estadual do Norte Fluminense Darcy Ribeiro - UENF, Rio de Janeiro.

Martínez, A. 2010. Estudio de la absorción y translocación del nitrógeno en cítricos en función del aporte estacional del abono nitrogenado, mediante la técnica de dilución isotópica. Tesis Doctorado Ingeniero Agrónomo, Instituto valenciano de investigaciones Agrarias, Valencia.

Mazzarino, M. 2002. Circulación de nutrientes en ecosistemas naturales: Conservación en la vegetación y dinámica en el suelo. XVIII Congreso Argentino de la Ciencia del Suelo. Asociación Argentina de la ciencia del suelo, Puerto Madryn, Abril de 2002, p12. 
Medina, A., García, S. y Schrevens, E. 2005. Annual Optimization and implementation of green waste compost applications in sustainable agriculture in the high tropics. Progress report VLIR-OI. 1(1).

Navarro, S. y Navarro, G. 2000. Química agrícola, el suelo y los elementos químicos esenciales para la vida vegetal. Ediciones Mundi-Prensa, Madrid, p183-218.

Rodríguez, E. 2002. Bases de conocimientos para generar modelos predictivos de respuesta a los fertilizantes nitrogenados en agroecosistemas cañeros. Tesis Maestro en Ciencias Agrarias. Universidad Agraria de la Habana (UNAH), La Habana.

Rodríguez, J. y Matus, F. 1989. Modelo simple para estimar el suministro de nitrógeno en el suelo. Revista Ciencia e Investigación Agraria 16:33-46.
Stanford, G y Smith, S. 1972. Nitrogen mineralization potentials of soils. Soil Science Society of American Proceedings 36(3):465-472.

Sikora, L. y Szmidt, R. 2004. Los compost como fuentes de nitrógeno, aportación a la mineralización y ventajas para la nutrición nitrogenada de las plantas. En: Stoffella, P. y Kahn, B. (Ed). Utilización de compost en los sistemas de cultivo hortícola. Ediciones Mundi-Prensa, Barcelona, p414.

Vernimmen, R., Verhoefa, H., Verstratenb, J., Bruijnzeela, L., Klompa, N., Zoomera, H. y Wartenbergh, P. 2007. Nitrogen mineralization, nitrification and denitrification potential in contrasting lowland rain forest types in Central Kalimantan, Indonesia. Soil Biology \& Biochemistry 39(12):2992-3003. 